\title{
Segre classes of tautological bundles on Hilbert schemes of surfaces
}

\author{
Claire Voisin
}

\begin{abstract}
We first give an alternative proof, based on a simple geometric argument, of a result of Marian, Oprea and Pandharipande on top Segre classes of the tautological bundles on Hilbert schemes of $K 3$ surfaces equipped with a line bundle. We then turn to the blowup of a $K 3$ surface at one point and establish vanishing results for the corresponding top Segre classes in a certain range. This determines, at least theoretically, all top Segre classes of tautological bundles for any pair $(\Sigma, H)$, where $H \in \operatorname{Pic} \Sigma$.
\end{abstract}

\section{Introduction}

Let $S$ be a smooth projective (or compact complex) surface. The Hilbert scheme $S^{[k]}$ is smooth projective (or compact complex) of dimension $2 k$. For any line bundle $H$ on $S$, we get an associated vector bundle $\mathcal{H}_{[k]}$ on $S^{[k]}$, whose fiber at a point $[Z] \in S^{[k]}$ is the vector space $H^{0}\left(H_{\mid Z}\right)$. If $S$ is a $K 3$ surface and $c_{1}(H)^{2}=2 g-2$, we set

$$
s_{k, g}:=\int_{S^{[k]}} s_{2 k}\left(\mathcal{H}_{[k]}\right),
$$

where $s_{i}\left(\mathcal{H}_{[k]}\right)$ is the $i$ th Segre class of $\mathcal{H}_{[k]}$. This integral is indeed a number which depends only on $k$ and $g$ (see Theorem 1.2). The following result is proved in [MOP17a].

THEOREM 1.1. One has $s_{k, g}=2^{k}\left(\begin{array}{c}g-2 k+1 \\ k\end{array}\right)$.

Here the binomial coefficient is defined for $k \geqslant 0$. It is always 1 for $k=0$, and the formula for $\left(\begin{array}{l}n \\ k\end{array}\right)$ for any $n$ is

$$
\left(\begin{array}{l}
n \\
k
\end{array}\right)=\frac{n(n-1) \cdots(n-k+1)}{k !} .
$$

In particular, we have $\left(\begin{array}{l}n \\ k\end{array}\right)=0$ if $n \geqslant 0$ and $n<k$. The theorem above thus gives, in particular, the vanishing

$$
s_{k, g}=0 \quad \text { when } g-2 k+1 \geqslant 0 \text { and } k>g-2 k+1 .
$$

The proof of this vanishing statement in [MOP17a] is rather involved; in Section 2, we will give a direct geometric proof, based on a small improvement of Lazarsfeld's arguments in [Laz86].

Received 30 August 2017, accepted in final form 9 November 2017.

2010 Mathematics Subject Classification 14N10 (primary), 14J99 (secondary).

Keywords: punctual Hilbert scheme, Segre classes, tautological bundles.

This journal is (c) Foundation Compositio Mathematica 2019. This article is distributed with Open Access under the terms of the Creative Commons Attribution Non-Commercial License, which permits non-commercial reuse, distribution, and reproduction in any medium, provided that the original work is properly cited. For commercial re-use, please contact the Foundation Compositio Mathematica.

The author acknowledges the support of Dr. Max Rössler, the Walter Haefner Foundation and the ETH Zurich Foundation. 


\section{SEgRe Classes of taUtological BUNDLES}

(We discovered after writing up the paper that this improvement had already been proved by Knutsen [Knu01].)

We will then show how the vanishing (1.1), even only in the smaller range $g=2 k-1$, $g=2 k$, implies Theorem 1.1. We simply use for this the following result, which is due to Tikhomirov [Tik94] (see also Ellingsrud-Göttsche-Lehn [EGL01] and Lehn [Leh99] for related statements).

TheOREm 1.2. The Segre numbers $\int_{S^{[k]}} s_{2 k}\left(\mathcal{H}_{[k]}\right)$ for a projective surface $S$ equipped with a line bundle $H$ depend only on the four numbers

$$
\pi=H \cdot K_{S}, \quad d=H^{2}, \quad \kappa=K_{S}^{2}, \quad e=c_{2}(S) .
$$

We will denote these Segre numbers by $s_{k}(d, \pi, \kappa, e)$. It follows from Theorem 1.2 that the numbers $s_{k, g}$ can be computed as well by considering a surface $\Sigma$ which is the disjoint union of a $K 3$ surface $S^{\prime}$, equipped with a line bundle $H^{\prime}$ of self-intersection $2(g-1)-2$, and an abelian surface $A$, equipped with a line bundle $\theta$ with $\theta^{2}=2$. We will show in Section 3 that the formula obtained by this observation (this is a particular case of (1.3) below), combined with the vanishing result (1.1), uniquely determine the numbers $b_{k}:=\int_{A^{[k]}} s_{2 k}\left(\theta_{[2 k]}\right)$ and finally the numbers $s_{k, g}$ for all $k, g$, knowing that $s_{1, g}=2 g-2, b_{0}=1, b_{1}=2$.

In Section 2, we will establish similar vanishing results for a $K 3$ surface $S$ blown up at one point. Let $\widetilde{S}$ be such a surface, and let $H=\tau^{*} L(-l E)$ with $2 g-2=L^{2}$, where $L$ generates Pic $S$.

THEOREM 1.3. For $k \geqslant 2$, one has the following vanishing for the Segre numbers $\tilde{s}_{k, g, l}:=$ $\int_{\widetilde{S}^{[k]}} s_{2 k}\left(\mathcal{H}_{[k]}\right)$ :

$$
\tilde{s}_{k, g, l}=0 \quad \text { for } \quad k=l, l+1 \quad \text { and } \quad g-\frac{l(l+1)}{2}=3 k-2 .
$$

Equivalently, $s_{k}(7(k-1), k-1,-1,25)=0$ and $s_{k}(7(k-1)+1, k,-1,25)=0$ for $k \geqslant 2$.

We will also prove that these vanishing statements together with Theorem 1.1 determine all Segre numbers $s_{k}(d, \pi, \kappa, e)$. We use for this the following complement to Theorem 1.2 (see [Leh99, EGL01]), obtained by observing that the Hilbert scheme $S^{[k]}$ of a disjoint union $S_{1} \sqcup S_{2}$ is the disjoint union for $l=0, \ldots, k$ of $S_{1}^{[l]} \times S_{2}^{[k-l]}$, while all the data $d, \pi, \kappa, e$ for the pairs $(\Sigma, H)$ are additive under disjoint unions $(S, L)=\left(S_{1}, L_{1}\right) \sqcup\left(S_{2}, L_{2}\right)$.

Lemma 1.4. With the notation $S_{d, \pi, \kappa, e}(z)=\sum_{k} s_{k}(d, \pi, \kappa, e) z^{k}$, one has

$$
S_{d, \pi, \kappa, e}(z)=S_{d_{1}, \pi_{1}, \kappa_{1}, e_{1}}(z) S_{d_{2}, \pi_{2}, \kappa_{2}, e_{2}}(z)
$$

with $d=d_{1}+d_{2}, \pi=\pi_{1}+\pi_{2}$ etc.

To conclude this introduction, we mention Lehn's conjecture [Leh99, Conjecture 4.9].

Conjecture 1.5. One has

$$
S_{d, \pi, \kappa, e}(z)=\frac{(1-w)^{a}(1-2 w)^{b}}{\left(1-6 w+6 w^{2}\right)^{c}},
$$

where $a=\pi-2 \kappa, b=d-2 \pi+\kappa+3 \chi, c=(d-\pi) / 2+\chi, \chi=(\kappa+e) / 12$ and the variable $w$ is related to $z$ by

$$
z=\frac{w(1-w)(1-2 w)^{4}}{\left(1-6 w+6 w^{2}\right)^{3}}
$$




\section{VOISIN}

This conjecture is proved in [MOP17a] for $K 3$ and, more generally, $K$-trivial surfaces, that is, for $\kappa=\pi=0$. Although we were not able to prove it in general, our results imply the following.

Corollary 1.6. Lehn's conjecture is equivalent to the fact that the development in power series in $z$ of the Lehn function $f_{d, \pi, \kappa, e}(z)$ defined as the right-hand side in (1.4) using the change of variables (1.5) has vanishing Taylor coefficient of order $k$ for $k \geqslant 2$ and for $e=25, \kappa=-1$ and $(d, \pi)=(7(k-1), k-1)$ or $(d, \pi)=(7(k-1)+1, k)$.

Shortly after this paper was written, Marian-Oprea-Pandharipande [MOP19] and SzenesVergne independently were able to check that the Lehn function satisfies the vanishing properties stated in Corollary 1.6, thus completing the proof of Lehn's conjecture.

Let us mention the following intriguing question: Lehn's conjecture (now a theorem) singles out the class of pairs $(S, H)$ with the following numerical properties:

$$
H^{2}=0, \quad H \cdot K_{S}=2 K^{2}=2 \chi\left(\mathcal{O}_{S}\right) .
$$

These conditions are indeed equivalent to the vanishing of the exponents $a, b$ and $c$ appearing in (1.4), so that for these pairs, one has the vanishing $s_{2 k}\left(\mathcal{H}_{[k]}\right)=0$. It would be nice to have a geometric proof of this.

\section{Geometric vanishing}

Let $S$ be a $K 3$ surface with Pic $S=\mathbb{Z} H$, where $H$ is an ample line bundle of self-intersection $2 g-2$. In this section, we give a geometric proof of the vanishing result (1.1) proved in [MOP17a].

Proposition 2.1. The Segre classes $s_{2 k}\left(\mathcal{H}_{[k]}\right)$ vanish in the range

$$
3 k-1>g>2 k-2 \text {. }
$$

In particular, $s_{k, 2 k}=0$ and $s_{k, 2 k-1}=0$ when $k \geqslant 2$.

Proof. Sections of $H$ provide sections of $\mathcal{H}_{[k]}$ or, equivalently, of the line bundle $\mathcal{O}_{\mathbf{P}\left(\mathcal{H}_{[k]}^{*}\right)}(1)$. In fact, all sections of $\mathcal{H}_{[k]}$ come from $H^{0}(S, H)$. As we are on a $K 3$ surface, $H^{0}(S, H)$ has dimension $g+1$. We thus have a rational map $\phi: \mathbf{P}\left(\mathcal{H}_{[k]}^{*}\right) \rightarrow \mathbf{P}^{g}$ such that $\phi^{*} \mathcal{O}_{\mathbf{P}^{g}}(1)=\mathcal{O}_{\mathbf{P}\left(\mathcal{H}_{[k]}^{*}\right)}(1)$. The top Segre class of $\mathcal{H}_{[k]}^{*}\left(\right.$ or $\left.\mathcal{H}_{[k]}\right)$ is the top self-intersection of $c_{1}\left(\mathcal{O}_{\mathbf{P}\left(\mathcal{H}_{[k]}^{*}\right)}(1)\right)$ on $\mathbf{P}\left(\mathcal{H}_{[k]}^{*}\right)$. We observe that the first inequality in (2.1) says that $\operatorname{dim} \mathbf{P}\left(\mathcal{H}_{[k]}^{*}\right)>\operatorname{dim} \mathbf{P}^{g}$, so the proposition is a consequence of the following lemma, which is a mild generalization of Lazarsfeld's result in [Laz86] saying that smooth curves in $|H|$ are Brill-Noether generic.

Lemma 2.2. If $g>2 k-2$, the vector bundle $\mathcal{H}_{[k]}$ is generated by the sections coming from $H^{0}(S, H)$.

Indeed, this last statement says that the rational map $\phi$ is actually a morphism, so that the top self-intersection of a line bundle pulled back via $\phi$ is 0 .

Proof of Lemma 2.2. This result had been already proved in [Knu01]. The proof is by contradiction. It is obtained by applying Lazarsfeld's arguments in [Laz86]. For the convenience of the reader and because Lazarsfeld considers only subschemes supported on smooth curves, we give the complete argument: If $z \in S^{[k]}$ is a point such that $H^{0}(S, H) \rightarrow \mathcal{H}_{[k], z}$ is not surjective, $z$ corresponds to a length $k$ subscheme $Z \subset S$ such that the restriction map $H^{0}(S, H) \rightarrow H^{0}\left(H_{\mid Z}\right)$ 


\section{SEgRe Classes of taUtological BUNDLES}

is not surjective, hence $H^{1}\left(S, \mathcal{I}_{Z}(H)\right) \neq 0$. By Serre duality, we thus have a nonzero class $e \in \operatorname{Ext}^{1}\left(\mathcal{I}_{Z}, H^{-1}\right)$, which provides a torsion-free rank 2 sheaf $\mathcal{E}$ fitting into an exact sequence

$$
0 \rightarrow H^{-1} \rightarrow \mathcal{E} \rightarrow \mathcal{I}_{Z} \rightarrow 0
$$

Note that the original Lazarsfeld argument deals with all subschemes which are locally complete intersection, for which $\mathcal{E}$ is locally free (assuming that $k$ is minimal). We have $c_{1}(\mathcal{E})=H^{-1}$ and $c_{2}(\mathcal{E})=k$. It thus follows that

$$
\begin{aligned}
\chi(\mathcal{E}, \mathcal{E}) & :=h^{0}(\operatorname{End}(\mathcal{E}))-\operatorname{dim} \operatorname{Ext}^{1}(\mathcal{E}, \mathcal{E})+\operatorname{dim} \operatorname{Ext}^{2}(\mathcal{E}, \mathcal{E})=4 \chi\left(\mathcal{O}_{S}\right)+c_{1}(\mathcal{E})^{2}-4 c_{2}(\mathcal{E}) \\
& =8+2 g-2-4 k .
\end{aligned}
$$

The second inequality in (2.1) thus gives

$$
\chi(\mathcal{E}, \mathcal{E})>2 .
$$

We thus conclude (applying Serre duality showing that $\operatorname{dim} \operatorname{Ext}^{2}(\mathcal{E}, \mathcal{E})=h^{0}(\operatorname{End}(\mathcal{E}))$ ) that $\mathcal{E}$ has an endomorphism $f: \mathcal{E} \rightarrow \mathcal{E}$ which is not proportional to the identity, hence can be assumed to be of generic rank 1 . Let $B$ be the line bundle defined as $\mathcal{F}^{* *}$, where $\mathcal{F}$ is the saturation of $\operatorname{Im} f$ in $\mathcal{E}$. The line bundle $B$ must be a power of $H$. The nonsplit exact sequence (2.2) shows that $\operatorname{Hom}\left(\mathcal{E}, H^{-1}\right)=0$ since $\operatorname{hom}\left(\mathcal{I}_{Z}, H^{-1}\right)=0$, so $B$ must be trivial or a positive power of $H$. It follows that $\mathcal{F}$ is equal to $H^{\otimes k} \otimes \mathcal{I}_{W}$ for some $k \geqslant 0$ and for some 0 -dimensional subscheme $W \subset Z$ (which can appear only where $\mathcal{E}$ is not locally free). As $H^{\otimes k} \otimes \mathcal{I}_{W}$ is not contained in $H^{-1}$, it must map nontrivially to $\mathcal{I}_{Z}$ via $f: \mathcal{E} \rightarrow \mathcal{I}_{Z}$, so that finally $k=0$ and $\mathcal{I}_{W} \subset \mathcal{I}_{Z}$. As $\mathcal{I}_{Z} \subset \mathcal{I}_{W}$ and $\operatorname{End}\left(\mathcal{I}_{Z}\right)=\mathbb{C} I d$, we conclude that, in fact, $f$ induces an isomorphism $\mathcal{I}_{W} \cong \mathcal{I}_{Z}$ and the sequence (2.2) is split, which gives a contradiction.

We note for later reference the following simple fact on which the proof of Proposition 2.1 rests. We will say that a line bundle $H$ on a variety $X$ is $k$-ample if the vector bundle $\mathcal{H}_{[k]}$ on $X^{[k]}$ is generated by its global sections; 1 -ample means that $H$ is generated by sections, and 2-ample means that $H$ is very ample.

Lemma 2.3. Let $\Sigma$ be a surface, $H$ a line bundle on $\Sigma$. Assume that $H$ is $k$-ample and $h^{0}(\Sigma, H)<$ $3 k$. Then $s_{2 k}\left(\mathcal{H}_{[k]}\right)=0$.

\section{Proof of Theorem 1.1}

We are going to prove Theorem 1.1 for $2 g-2 \geqslant 0$, that is, $g \geqslant 1$, by induction on $g$. The case where $g$ is nonpositive works similarly, by induction on $-g$. Let $S^{\prime}$ be a $K 3$ surface equipped with a line bundle $H^{\prime}$ such that $c_{1}\left(H^{\prime}\right)^{2}=2(g-1)-2$. Let $A$ be an abelian surface with a principal polarization $\theta$, so that $c_{1}(\theta)^{2}=2$. The surface $\Sigma=S^{\prime} \sqcup A$ equipped with the line bundle $H_{\Sigma}$ which is equal to $H^{\prime}$ on $S^{\prime}$ and $\theta$ on $A$ has the same characteristic numbers as our original pair $(S, H)$, where $S$ is a $K 3$ surface and $H$ is a polarization with self-intersection $2 g-2$. On the other hand, $\Sigma^{[k]}$ is the disjoint union

$$
\Sigma^{[k]}=\bigsqcup_{l=0}^{l=k} S^{\prime[k-l]} \times A^{[l]},
$$

and on each summand $S^{\prime[k-l]} \times A^{[l]}$, the vector bundle $H_{\Sigma,[k]}$ equals $\operatorname{pr}_{1}^{*} H_{[k-l]}^{\prime} \oplus \operatorname{pr}_{2}^{*} \theta_{[l]}$. We thus conclude that we have the following formula, where $b_{l}:=\int_{A^{[l]}} s_{2 l}\left(\theta_{[2 l]}\right)$ (this is a particular case 


\section{VOISIN}

of $(1.3))$ :

$$
s_{k, g}=\sum_{l=0}^{l=k} b_{l} s_{k-l, g-1} .
$$

Corollary 3.1. The numbers $s_{k, g}$ for $g \geqslant 1$ are fully determined by the numbers $b_{l}$ for $0 \leqslant l \leqslant k$ and the numbers $s_{l, 1}$ for $l \leqslant k$ and $s_{1, g}$ for $g \geqslant 1$ (or $s_{0, g}$ ).

Remark 3.2. We have $b_{0}=1$ and $b_{1}=2$ and, similarly, $s_{0, g}=1$ and $s_{1, g}=2 g-2$.

Lemma 3.3. Suppose that the numbers $b_{l}$, for $0 \leqslant l \leqslant k-1$, and the numbers $s_{l, 1}$, for $0 \leqslant l \leqslant k-1$, are given, with $b_{0}=1$ and $b_{1}=2$. Then the numbers $s_{k, 1}$ and $b_{k}$ are determined by the conditions $b_{0}=1$ and $b_{1}=2$, equation (3.1) and the vanishing equations

$$
s_{k, 2 k}=0, \quad s_{k, 2 k-1}=0
$$

for $k \geqslant 2$ proved in Proposition 2.1.

Proof. Indeed, by Corollary 3.1, all the numbers $s_{l, g^{\prime}}$ for $g^{\prime} \leqslant g-1$ and $l \leqslant k-1$ are determined by $b_{l}$ for $0 \leqslant l \leqslant k-1$ and $s_{l, 1}$ for $0 \leqslant l \leqslant k-1$. We thus can write (3.1) as

$$
\begin{aligned}
s_{k, g} & =s_{k, g-1}+(\cdots)+b_{k}, \\
s_{k, g-1} & =s_{k, g-2}+(\cdots)+b_{k},
\end{aligned}
$$

where the expressions $(\cdots)$ in the middle are determined by $b_{l}$ for $0 \leqslant l \leqslant k-1$ and $s_{l, 1}$ for $0 \leqslant l \leqslant k-1$. Combining these equations, we get

$$
\begin{aligned}
s_{k, 2 k} & =s_{k, 1}+(\cdots)+(2 k-1) b_{k}, \\
s_{k, 2 k-1} & =s_{k, 1}+(\cdots)+(2 k-2) b_{k} ;
\end{aligned}
$$

hence, we can see the equations $s_{k, 2 k}=0$ and $s_{k, 2 k-1}=0$ as a system of two affine equations in the two variables $s_{k, 1}$ and $b_{k}$, whose linear part is invertible and whose constants are determined by $b_{l}$ for $0 \leqslant l \leqslant k-1$ and $s_{l, 1}$ for $0 \leqslant l \leqslant k-1$. The numbers $s_{k, 1}$ and $b_{k}$ are thus uniquely determined by these equations and the numbers $b_{l}$ for $0 \leqslant l \leqslant k-1$ and $s_{l, 1}$ for $0 \leqslant l \leqslant k-1$.

Corollary 3.4. There exist unique sequences of numbers $s_{k, g}$, for $k \geqslant 0$ and $g \geqslant 1$, and $b_{l}$, for $l \geqslant 0$, satisfying

(i) $b_{0}=1, b_{1}=2$,

(ii) $s_{0, g}=1, s_{1, g}=2 g-2$,

(iii) $s_{k, 2 k}=0, s_{k, 2 k-1}=0$ for $k \geqslant 2$,

(iv) $s_{k, g}=\sum_{l=0}^{l=k} b_{l} s_{k-l, g-1}$.

Proof of Theorem 1.1. The numbers $s_{k, g}^{\prime}:=2^{k}\left(\begin{array}{c}g-2 k+1 \\ k\end{array}\right)$ satisfy the vanishings $s_{k, 2 k}^{\prime}=0$ and $s_{k, 2 k-1}^{\prime}=0$ for $k \geqslant 2$, that is, condition (iii) of Corollary 3.4. They also satisfy the condition $s_{1, g}^{\prime}=2 g-2$, that is, condition (ii) of Corollary 3.4. In order to show that $s_{k, g}=s_{k, g}^{\prime}$, it suffices by Corollary 3.4 to show that they also satisfy condition (iv) for adequate numbers $b_{l}^{\prime}$, which is proved in Lemma 3.5 below.

Lemma 3.5. There exist numbers $b_{l}^{\prime}$ for $l \geqslant 0$ with $b_{0}^{\prime}=1$ and $b_{1}^{\prime}=2$ such that for any $g \geqslant 1$,

$$
s_{k, g}^{\prime}=\sum_{l=0}^{k} b_{l}^{\prime} s_{k-l, g-1}^{\prime} .
$$




\section{SEgRe Classes of taUtological BUNDLES}

Proof. We observe that as a function of $g$, the polynomial $s_{k, g}^{\prime}$ is of degree exactly $k$, with leading coefficient $2^{k}$. Hence, the $s_{l, g}^{\prime}$ for $0 \leqslant l \leqslant k-1$ form a basis of the space of polynomials of degree $k-1$, and for $k$ fixed, there exist uniquely defined numbers $b_{l, k}^{\prime}$ for $l=0, \ldots, k$, with $b_{0, k}^{\prime}=1$, such that for any $g$,

$$
s_{k, g}^{\prime}=\sum_{l=0}^{k} b_{l, k}^{\prime} s_{k-l, g-1}^{\prime} .
$$

Let us prove that $b_{l, k}^{\prime}=b_{l, k-1}^{\prime}$ for $l \leqslant k-1$. We have

$$
\left(\begin{array}{c}
g-2 k+1 \\
k
\end{array}\right)=\left(\begin{array}{c}
g-2 k \\
k
\end{array}\right)+\left(\begin{array}{c}
g-2 k \\
k-1
\end{array}\right)
$$

that is,

$$
2 s_{k-1, g-3}^{\prime}=s_{k, g}^{\prime}-s_{k, g-1}^{\prime},
$$

with the convention that $s_{k, g}^{\prime}=0$ for $k<0$. It follows by the definition of $b_{l, k}^{\prime}$ that

$$
2 s_{k-1, g-3}^{\prime}=\sum_{l=0}^{k} b_{l, k}^{\prime} s_{k-l, g-1}^{\prime}-\sum_{l=0}^{k} b_{l, k}^{\prime} s_{k-l, g-2}^{\prime}=\sum_{l=0}^{k} b_{l, k}^{\prime}\left(s_{k-l, g-1}^{\prime}-s_{k-l, g-2}^{\prime}\right),
$$

which gives, by applying (3.6) again to each term on the right-hand side,

$$
2 s_{k-1, g-3}^{\prime}=2 \sum_{l=0}^{k} b_{l, k}^{\prime} s_{k-l-1, g-4}^{\prime}=2 \sum_{l=0}^{k-1} b_{l, k}^{\prime} s_{k-l-1, g-4}^{\prime} .
$$

By the definition of $b_{l, k-1}^{\prime}$, this provides $b_{l, k}^{\prime}=b_{l, k-1}^{\prime}$.

\section{Further geometric vanishing results}

We discuss in this section similar geometric vanishing results for the Segre classes on the blow-up of a $K 3$ surface at one point. The setting is thus the following: $S$ is a $K 3$ surface with Pic $S=\mathbb{Z} L$, where $L^{2}=2 g-2$ and $x \in S$ is a point. The surface $\widetilde{S}$ is the blow-up $\tau: \widetilde{S} \rightarrow S$ of $S$ at $x$ with exceptional curve $E$, and $H:=\tau^{*} L(-l E) \in \operatorname{Pic} \widetilde{S}$ for some positive integer $l$. Our main goal is to discuss the analogue of Lemma 2.2 in this context. Note that when $H$ is very ample, the curve $E$ has degree $l$ in the embedding given by $|H|$, so that the vector bundle $\mathcal{H}_{[k]}$ can be generated by sections only when $k \leqslant l+1$.

To start with, we have the following result.

Proposition 4.1. Let $S$ be a $K 3$ surface with Picard group generated by $L$, where $L^{2}=2 g-2$. Let $\tau: \widetilde{S} \rightarrow S$ be the blow-up at a point $x \in S$. Then, for $H=\tau^{*} L(-l E)$, if

$$
4+2 g>(l+1)^{2},
$$

one has $H^{1}(\widetilde{S}, H)=0$. It follows that $h^{0}(\widetilde{S}, H)=g+1-l(l+1) / 2$.

Proof. We argue by contradiction. The proof follows Reider's [Rei88] and Lazarsfeld's [Laz86] methods. Assume $H^{1}(\widetilde{S}, H) \neq 0$. Then, by Serre duality, $\operatorname{Ext}^{1}\left(H, \mathcal{O}_{\widetilde{S}}(E)\right) \neq 0$, which provides a rank 2 vector bundle $\mathcal{E}$ on $\widetilde{S}$ which fits in an exact sequence

$$
0 \rightarrow \tau^{*} L^{-1}((l+1) E) \rightarrow \mathcal{E} \rightarrow \mathcal{O}_{\widetilde{S}} \rightarrow 0 .
$$

The fact that the extension class of $(4.2)$ is not trivial translates into $h^{0}(\widetilde{S}, \mathcal{E})=0$. We have 


\section{VOISIN}

$c_{2}(\mathcal{E})=0$ and $c_{1}(\mathcal{E})^{2}=2 g-2-(l+1)^{2}$, so that (4.1) gives the inequality

$$
\chi(\text { End } \mathcal{E})=8+c_{1}(\mathcal{E})^{2}-4 c_{2}(\mathcal{E})>2 .
$$

It follows that $h^{0}(\widetilde{S}$, End $\mathcal{E})+h^{0}(\widetilde{S}$, End $\mathcal{E}(E))>2$, hence $h^{0}(\widetilde{S}$, End $\mathcal{E}(E))>1$. Thus there exists a $\phi \in \operatorname{Hom}(\mathcal{E}, \mathcal{E}(E))$ which is not proportional to the identity. The characteristic polynomial of $\phi$ has its trace in $H^{0}\left(\widetilde{S}, \mathcal{O}_{\widetilde{S}}(E)\right)=H^{0}\left(\widetilde{S}, \mathcal{O}_{\widetilde{S}}\right)$ and determinant in $H^{0}\left(\widetilde{S}, \mathcal{O}_{\widetilde{S}}(2 E)\right)=H^{0}\left(\widetilde{S}, \mathcal{O}_{\widetilde{S}}\right)$. It is thus a polynomial with coefficients in $\mathbb{C}$ and has a root $\lambda$. Replacing $\phi$ by $\phi-\lambda \mathrm{Id}_{\mathcal{E}}$ (where we see $\operatorname{Id}_{\mathcal{E}}$ as an element of $\operatorname{Hom}(\mathcal{E}, \mathcal{E}(E))$ ), we can in fact assume that $\phi$ is generically of rank 1 . Let $A=\operatorname{Ker} \phi \subset \mathcal{E}$. We have $A=\tau^{*} L^{\alpha}(\beta E)$, and $\mathcal{E}$ fits in an exact sequence

$$
0 \rightarrow A \rightarrow \mathcal{E} \rightarrow B \otimes \mathcal{I}_{W} \rightarrow 0,
$$

where $B$ is the line bundle $\tau^{*} L^{-1-\alpha}((l+1-\beta) E)$. As $B=\operatorname{Im} \phi$, we have $B \hookrightarrow \mathcal{E}(E)$. From the exact sequence (4.2), we immediately conclude that $\alpha \leqslant 0$ and $(-1-\alpha) \leqslant 0$, so that $\alpha=0$ or $\alpha=-1$.

First assume $\alpha=0$. Then as $h^{0}(\widetilde{S}, \mathcal{E})=0$, we conclude that $\beta<0$, hence $l+1-\beta>0$. Then (4.3) gives

$$
c_{2}(\mathcal{E})=A \cdot B+\operatorname{deg} W \geqslant-\beta(l+1-\beta)>0,
$$

which gives a contradiction.

In the remaining case $\alpha=-1$, we conclude that $B=\mathcal{O}_{\widetilde{S}}((l+1-\beta) E)$, so that we have a nonzero morphism $\mathcal{O}_{\widetilde{S}}((l-\beta) E) \rightarrow \mathcal{E}$. This provides a line bundle $A^{\prime} \subset \mathcal{E}$ defined as the saturation of the image of this morphism, and we know that $A^{\prime}=\tau^{*} L^{\alpha^{\prime}}\left(\beta^{\prime} E\right)$ with $\alpha^{\prime} \geqslant 0$. We can then apply the previous argument with $A$ replaced with $A^{\prime}$, getting a contradiction.

Pushing forward the arguments above, we now prove the following result.

Theorem 4.2. Let $S$ be a general $K 3$ surface with Picard group generated by $L$ and $x \in S$ a general point. Then for $k \geqslant 2$, the line bundle $H=\tau^{*} L(-l E)$ is $k$-ample on $\widetilde{S}$ for $k=l$ or $k=l+1$, and $g-l(l+1) / 2=3 k-2$.

Remark 4.3. When $g-l(l+1) / 2=3 k-2$ with $k=l$ or $k=l+1$, one has, for $l>0$,

$$
4+2 g=l(l+1)+6 k \geqslant(l+7) l>(l+1)^{2},
$$

so that Proposition 4.1 applies, which gives $H^{1}(\widetilde{S}, H)=0$ and $h^{0}(\widetilde{S}, H)=g+1-l(l+1) / 2=$ $3 k-1$.

Proof of Theorem 4.2. With the assumptions of Theorem 4.2, assume that $H$ is not $k$-ample. Therefore, there exists a 0 -dimensional subscheme $Z \subset \widetilde{S}$ of length $k$ such that $H^{1}\left(\widetilde{S}, H \otimes \mathcal{I}_{Z}\right) \neq 0$. Using the duality $H^{1}\left(\widetilde{S}, H \otimes \mathcal{I}_{Z}\right)^{*}=\operatorname{Ext}^{1}\left(\mathcal{I}_{Z},-H+E\right)$, this provides us with a rank 2 torsion-free sheaf $\mathcal{E}$ on $\widetilde{S}$ fitting in an exact sequence

$$
0 \rightarrow \tau^{*} L^{-1}((l+1) E) \rightarrow \mathcal{E} \rightarrow \mathcal{I}_{Z} \rightarrow 0 .
$$

The numerical invariants of $\mathcal{E}$ are given by

$$
c_{2}(\mathcal{E})=k, \quad c_{1}(\mathcal{E})^{2}=2 g-2-(l+1)^{2},
$$

from which we conclude that

$$
\chi(\mathcal{E}, \mathcal{E})=8+2 g-2-(l+1)^{2}-4 k ;
$$

hence,

$$
h^{0}(\text { End } \mathcal{E})+h^{0}(\text { End } \mathcal{E}(E)) \geqslant 8+2 g-2-(l+1)^{2}-4 k \text {. }
$$




\section{SEgRe Classes of taUtological BUNDLES}

By assumption, $g-l(l+1) / 2=3 k-2$, so $2 g-2-(l+1)^{2}=6 k-6-(l+1)$ and $(4.5)$ gives

$$
2 h^{0}(\text { End } \mathcal{E}(E)) \geqslant 2+2 k-(l+1) ;
$$

hence, $2 h^{0}($ End $\mathcal{E}(E))>2$ because $k \geqslant 2$ and $k=l$ or $k=l+1$. Thus, there exists a morphism

$$
\phi: \mathcal{E} \rightarrow \mathcal{E}(E)
$$

which is not proportional to the identity. As before, we can even assume that $\phi$ is generically of rank 1 . One difference with the previous situation is the fact that $\mathcal{E}$ is not necessarily locally free, and furthermore $c_{2}(\mathcal{E}) \neq 0$. The kernel of $\phi$ and its image are torsion free of rank 1 , hence are of the form $A \otimes \mathcal{I}_{W}$ and $B \otimes \mathcal{I}_{W^{\prime}}$ for some line bundles $A$ and $B$ on $\widetilde{S}$ which are of the form

$$
A=\tau^{*} L^{\alpha}(\beta E), \quad B=\tau^{*} L^{-1-\alpha}((l+1-\beta) E) .
$$

As before, we must have $\alpha \leqslant 0$ and $-1-\alpha \leqslant 0$ because $B$ injects into $\mathcal{E}(E)$. Hence, we conclude that $\alpha=0$ or $\alpha=-1$.

Case (i). If $\alpha=0$, then we have a nonzero morphism $\mathcal{O}(\beta E) \otimes \mathcal{I}_{W} \rightarrow \mathcal{I}_{Z}$. It follows that $\beta \leqslant 0$. If $\beta=0$, this says that $\mathcal{I}_{W} \subset \mathcal{I}_{Z}$ and that the extension class of (4.4) vanishes in

$$
\operatorname{Ext}^{1}\left(\mathcal{I}_{W}, \tau^{*} L^{-1}((l+1) E)\right) .
$$

But the restriction map

$$
\operatorname{Ext}^{1}\left(\mathcal{I}_{Z}, \tau^{*} L^{-1}((l+1) E)\right) \rightarrow \operatorname{Ext}^{1}\left(\mathcal{I}_{W}, \tau^{*} L^{-1}((l+1) E)\right)
$$

is injective as it is dual to the map $H^{1}\left(\widetilde{S}, \mathcal{I}_{W}(H)\right) \rightarrow H^{1}\left(\widetilde{S}, \mathcal{I}_{Z}(H)\right)$, which is surjective. Indeed, the spaces are respective quotients of $H^{0}\left(H_{\mid W}\right)$ and $H^{0}\left(H_{\mid Z}\right)$ by Proposition 4.1, which applies in our case as noted in Remark 4.3. So we conclude that $\beta<0$. We now compute $c_{2}(\mathcal{E})$ using the exact sequence

$$
0 \rightarrow A \otimes \mathcal{I}_{W} \rightarrow \mathcal{E} \rightarrow B \otimes \mathcal{I}_{W^{\prime}} \rightarrow 0
$$

with $A=\mathcal{O}(\beta E)$ and $B=\tau^{*} L^{-1}((l+1-\beta) E)$. This gives

$$
c_{2}(\mathcal{E})=\operatorname{deg} W+\operatorname{deg} W^{\prime}-\beta(l+1-\beta) \geqslant-\beta(l+1-\beta) \geqslant l+2 .
$$

This contradicts $c_{2}(\mathcal{E})=k \leqslant l+1$.

Case (ii). If $\alpha=-1$, then we use instead the inclusion $B \otimes \mathcal{I}_{W^{\prime}} \subset \mathcal{E}(E)$, with $B=\mathcal{O}((l+$ $1-\beta) E)$, and argue exactly as before.

We deduce the following vanishing for the Segre numbers $s_{k}(d, \pi, \kappa, e)$, defined as the integral $\int_{\Sigma^{[k]}} s_{2 k}\left(\mathcal{H}_{[k]}\right)$ for any pair $(\Sigma, H)$, where $\Sigma$ is a smooth compact surface, and

$$
d=H^{2}, \quad \pi=H \cdot c_{1}\left(K_{\Sigma}\right), \quad \kappa=c_{1}(\Sigma)^{2}, \quad e=c_{2}(\Sigma) .
$$

Corollary 4.4 (cf. Theorem 1.3). One has the following vanishing for $s_{k}(d, \pi,-1,25)$ :

$$
s_{k}(7(k-1), k-1,-1,25)=0, \quad s_{k}(7(k-1)+1, k,-1,25)=0
$$

for $k \geqslant 2$.

Proof. Take for $\Sigma$ the blow-up of a $K 3$ surface at a point, so $\kappa=-1$ and $e=25$. Furthermore, assuming Pic $S=\mathbb{Z} L$ with $L^{2}=2 g-2$, and letting $H=\tau^{*} L(-l E)$ as above, we have

$$
d=H^{2}=2 g-2-l^{2}, \quad \pi=H \cdot c_{1}\left(K_{\Sigma}\right)=l .
$$




\section{VOISIN}

We consider the cases where

$$
g-\frac{l(l+1)}{2}=3 k-2
$$

with (i) $k=l+1$ or (ii) $k=l$.

Using (4.7), in case (i), equation (4.8) gives $d=7(k-1)$ and $\pi=k-1$, and in case (ii), it gives $d=7(k-1)+1$ and $\pi=k$, so that we are actually computing $s_{k}(7(k-1), k-1,-1,25)$ in case (i) and $s_{k}(7(k-1)+1, k,-1,25)$ in case (ii). Remark 4.3 says that assuming (4.8),

$$
H^{1}(\widetilde{S}, H)=0, \quad h^{0}(\widetilde{S}, H)=3 k-1
$$

in cases (i) and (ii). Theorem 4.2 says that under the same assumption, $H$ is $k$-ample on $\widetilde{S}$. Lemma 2.3 thus applies and gives $s_{2 k}\left(\mathcal{H}_{[k]}\right)=0$ in both cases, which is exactly (4.6).

Remark 4.5. In [Leh99, Section 4], Lehn gives the explicit polynomial formulas for $2 ! s_{2}, \ldots, 5 ! s_{5}$ as polynomial functions of $d, \pi, \kappa, e$ with huge integral coefficients. For example

$$
\begin{aligned}
5 ! s_{5}= & d^{5}-100 d^{4}+d^{3}(3740+10 e-50 \pi-10 \kappa) \\
& -d^{2}(62000-3420 \pi+700 e-860 \kappa)+d\left(384384+15 e^{2}+15960 e-30 e \kappa\right. \\
& \left.-150 \pi e+15 \kappa^{2}+150 \kappa \pi-75610 \pi-24340 \kappa+375 \pi^{2}\right)-400 e^{2}-117120 e \\
& +3920 \pi e+960 \kappa e+226560 \kappa-4720 \kappa \pi-560 \kappa^{2}+530880 \pi-9600 \pi^{2} .
\end{aligned}
$$

It is pleasant to check the vanishing statements (4.6) for $k=2, \ldots, 5$ using these formulas. For $k=5$, one just has to plug the values $e=25, \kappa=-1, d=28$ and $\pi=4$, or $e=25, \kappa=-1$, $d=29$ and $\pi=5$ into (4.9).

We conclude this note by showing that all the Segre numbers are formally determined by the above results and formula (1.3).

Proposition 4.6. The vanishings (4.6) together with the data of the numbers $s_{k}(d, 0,0,24)$ and $s_{k}(d, 0,0,0)$ determine all numbers $s_{k}(d, \pi, \kappa, e)$.

Note that for $d=2 g-2$, the number $s_{k}(d, 0,0,24)$ is the number $s_{k, g}$ of the introduction, and these numbers are given by Marian-Oprea-Pandharipande's Theorem 1.1. For $d$ even, the numbers $s_{k}(d, 0,0,0)$ correspond to the Segre classes of tautological sheaves on Hilbert schemes of abelian surfaces equipped with a line bundle of self-intersection $d$. They are fully determined, by multiplicativity, by the case of self-intersection 2 , where one gets the numbers $b_{k}^{\prime}$ appearing in our proof of Theorem 1.1.

Proof of Proposition 4.6. According to [Leh99, EGL01], and as follows from (1.3), the generating series $S_{d, \pi, \kappa, e}(z)=\sum_{k} s_{k}(d, \pi, \kappa, e) z^{k}$ is of the form

$$
S_{d, \pi, \kappa, e}(z)=A(z)^{d} B(z)^{e} C(z)^{\pi} D(z)^{\kappa}
$$

for power series $A, B, C, D$ with 0 th order coefficient equal to 1 . Theorem 1.1 determines the series $A(z)$ and $B(z)$. We thus only have to determine $C(z)$ and $D(z)$. The degree 1 coefficients of the power series $C(z)$ and $D(z)$ are immediate to compute as $s_{1}=d$. We now assume that the coefficients of the power series $C(z)$ and $D(z)$ are computed up to degree $k-1$. The degree $k$ coefficient of $S_{d, \pi, \kappa, e}(z)=A(z)^{d} B(z)^{e} C(z)^{\pi} D(z)^{\kappa}$ is of the form $\pi C_{k}+\kappa D_{k}+\nu$, where $\nu$ is determined by $d, e, \pi, \kappa$, the coefficients of $A$ and $B$, and the coefficients of order at most $k-1$ of $C$ and $D$. The vanishings (4.6) thus give the equations

$$
0=(k-1) C_{k}-D_{k}+\nu, \quad 0=k C_{k}-D_{k}+\nu^{\prime},
$$




\section{SEgRe Classes of taUtological BUNDLES}

which obviously determines $C_{k}$ and $D_{k}$ as functions of $\nu$ and $\nu^{\prime}$.

We finally prove Corollary 1.6 of the introduction.

Proof of Corollary 1.6. Let $f_{d, \pi, \kappa, e}(z)$ be the Lehn function introduced in Conjecture 1.5. As Lehn's conjecture is proved by [MOP17a] for $\pi=\kappa=0$ (the $K$-trivial case), the coefficients $f_{k, d, \pi, \kappa, e}$ of the Taylor expansion of $f_{d, \pi, \kappa, e}$ in $z$ (not $w$ ) are the Segre numbers $s_{k}(d, 0,0, e)$ when $\pi=0$ and $\kappa=0$. If, furthermore, they satisfy the vanishings $f_{k, d, \pi, \kappa, e}=0$ for $e=25, \kappa=-1$ and $d=7(k-1), \pi=k-1$ or $d=7(k-1)+1, \pi=k$, the proof of Proposition 4.6 shows that $f_{k, d, \pi, \kappa, e}=s_{k}(d, \pi, \kappa, e)$ for all $k, d, \pi, \kappa, e$ as, by definition, $f_{d, \pi, \kappa, e}$ has the same multiplicative form (4.10) as $S_{d, \pi, \kappa, e}$.

\section{ACKNOWLEDGEMEnTS}

I thank Rahul Pandharipande for discussions and, in particular, for suggesting, after I had given a geometric proof of the vanishings (1.1) on $K 3$ surfaces, to look at surfaces other than $K 3$ 's. I thank Olivier Debarre for indicating the reference [Knu01]. This work was done during my stay at ETH-ITS.

\section{REFERENCES}

EGL01 G. Ellingsrud, L. Göttsche and M. Lehn, On the cobordism class of the Hilbert scheme of a surface, J. Algebraic Geom. 10 (2001), no. 1, 81-100.

Knu01 A. Knutsen, On kth-order embeddings of K3 surfaces and Enriques surfaces, Manuscripta Math. 104 (2001), 211-237; doi:10.1007/s002290170040.

Laz86 R. Lazarsfeld, Brill-Noether-Petri without degenerations, J. Differential Geom. 23 (1986), no. 3, 299-307; doi:10.4310/jdg/1214440116.

Leh99 M. Lehn, Chern classes of tautological sheaves on Hilbert schemes of points on surfaces, Invent. Math. 136 (1999), no. 1, 157-207; doi:10.1007/s002220050307.

MOP17a A. Marian, D. Oprea and R. Pandharipande, Segre classes and Hilbert schemes of points, Ann. Sci. Éc. Norm. Supér. (4) 50 (2017), no. 1, 239-267; doi:10.24033/asens . 2320.

MOP19 , The combinatorics of Lehn's conjecture, J. Math. Soc. Japan, published online on 4 October 2018, doi:10.2969/jmsj/78747874, to appear in print.

Rei88 I. Reider, Vector bundles of rank 2 and linear systems on algebraic surfaces, Ann. of Math. $\mathbf{1 2 7}$ (1988), no. 2, 309-316; doi:10.2307/2007055.

Tik94 A.S. Tikhomirov, Standard bundles on a Hilbert scheme of points on a surface, Algebraic Geometry and its Applications (Yaroslavl', 1992), Aspects Math., vol. E25 (Friedr. Vieweg, Braunschweig, 1994), 183-203; doi:10.1007/978-3-322-99342-7_16.

Claire Voisin claire.voisin@imj-prg.fr

Collège de France, 3 rue d'Ulm, 75005 Paris, France 